\title{
INDEX REALIZATION FOR AUTOMORPHISMS OF FREE GROUPS
}

\author{
THIERRY COULBOIS AND MARTIN LUSTIG
}

\begin{abstract}
For any surface $\Sigma$ of genus $g \geq 1$ and (essentially) any collection of positive integers $i_{1}, i_{2}, \ldots, i_{\ell}$ with $i_{1}+\cdots+i_{\ell}=4 g-4$ Masur and Smillie 10 have shown that there exists a pseudo-Anosov homeomorphism $h: \Sigma \rightarrow \Sigma$ with precisely $\ell$ singularities $S_{1}, \ldots, S_{\ell}$ in its stable foliation $\mathcal{L}$, such that $\mathcal{L}$ has precisely $i_{k}+2$ separatrices raying out from each $S_{k}$.

In this paper we prove the analogue of this result for automorphisms of a free group $F_{N}$, where "pseudo-Anosov homeomorphism" is replaced by "fully irreducible automorphism" and the Gauss-Bonnet equality $i_{1}+$ $\cdots+i_{\ell}=4 g-4$ is replaced by the index inequality $i_{1}+\cdots+i_{\ell} \leq 2 N-2$ from [4].
\end{abstract}

\section{INTRODUCTION}

In 4 for every automorphism $\Phi \in \operatorname{Aut}\left(F_{N}\right)$ of a non-abelian free group $F_{N}$ of finite rank $N \geq 2$ an index $\operatorname{ind}(\Phi)$ has been defined, which counts in a natural way attracting fixed points at the Gromov boundary $\partial F_{N}$ and the rank of the fixed subgroup $\operatorname{Fix}(\Phi)$ of $\Phi$. If $\operatorname{Fix}(\Phi)=\{1\}$, then $2 \operatorname{ind}(\Phi)+2$ is simply the number of attractors of the homeomorphism $\partial \Phi: \partial F_{N} \rightarrow \partial F_{N}$ induced by $\Phi$.

As main result of [4] the index inequality

$$
\operatorname{ind}(\Phi) \leq N-1
$$

has been proved, which strengthens the celebrated Scott conjecture, proved in [1], and also extends some well known consequences of Nielsen-Thurston theory for surface homeomorphisms to free group automorphisms, in particular after passing to the stable index $\operatorname{ind}_{\text {stab }}(\varphi)$ of the associated outer automorphism $\varphi$, defined below in (1.1) as sum of ind $\left(\Phi_{k}\right)$ for suitable representatives $\Phi_{k}$ of a positive power of $\varphi$.

The main difference to surface homemorphisms, however, where the analog indices always sum up to give via Gauss-Bonnet the maximal possible value postulated in (1.1), is that $\operatorname{ind}_{s t a b}(\varphi)$ may well be strictly smaller than $N-1$.

1991 Mathematics Subject Classification. 20E05, 20E08, 20F65, 57R30.

Key words and phrases. Free group automorphisms, Train tracks, Index realization, Gate structure. 
Ever since it has been an open question which precise value of $\left\{\frac{1}{2}, 1, \frac{3}{2}, \ldots\right.$, $\left.\frac{2 N-3}{2}, N-1\right\}$ can be realized as stable index $\operatorname{ind}_{s t a b}(\varphi)$ by some $\varphi \in \operatorname{Out}\left(F_{N}\right)$, in particular if one restricts to automorphisms $\varphi$ of $F_{N}$ which are irreducible with irreducible powers (iwip), also called fully irreducible (see Section 2).

For any given $\varphi \in \operatorname{Out}\left(F_{N}\right)$ its representatives $\Phi_{k} \in \operatorname{Aut}\left(F_{N}\right)$ are partitioned into isogredience classes, where isogredient automorphisms are conjugate by inner automorphisms and hence have conjugate $\partial F_{N}$-dynamics and thus equal indices. It follows from the results of 4 that any $\Phi_{k}$ has a positive power $\Phi_{k}^{m_{k}}$ for which (as well as for all of its powers) the fixed subgroup and the number of attracting fixed points on $\partial F_{N}$ is maximal; the index of $\Phi_{k}^{m_{k}}$ will be called the stable index of $\Phi_{k}$ and denoted by $\operatorname{ind}_{s t a b} \Phi_{k}$.

The stable index list of $\varphi$ is defined as the longest sequence (up to permutation) of positive indices $\operatorname{ind}_{s t a b}\left(\Phi_{1}\right), \operatorname{ind}_{s t a b}\left(\Phi_{2}\right), \ldots, \operatorname{ind}_{s t a b}\left(\Phi_{\ell}\right)$, given by pairwise non-isogredient representatives $\Phi_{k}$ of some power $\varphi^{t}$, for any fixed $t \geq 1$. The inequalities

$$
\frac{1}{2} \leq \operatorname{ind}_{s t a b} \varphi:=\sum_{k=1}^{\ell} \operatorname{ind}_{s t a b} \Phi_{k} \leq N-1
$$

have been shown in [4]. Handel and Mosher [5, Question 6 in §1.5] have asked explicitly which such values are realized as stable index list by iwip automorphisms of $F_{N}$. We denote such a (potential) index list by $\left[j_{1}, \ldots, j_{\ell}\right]$, where the $j_{k}$ are usually given in decreasing order.

For the "maximal" case, i.e. $\operatorname{ind}_{\text {stab }}(\varphi)=N-1$, an almost complete answer to this question has been given by Masur and Smillie [10]: For $N \geq 3$ any list $\left[j_{1}, j_{2}, \ldots, j_{\ell}\right]$ of positive $j_{k} \in \frac{1}{2} \mathbb{Z}$, with $\sum j_{k}=N-1$ (other than the single exceptional case $\left[\frac{3}{2}, \frac{3}{2}\right]$ for $N=4$, see Section 7 below), can be realized as index list of an iwip automorphism which is geometric, i.e. $\varphi$ is induced by a pseudo-Anosov homeomorphism of a surface with one boundary component. On the other hand, if $\operatorname{ind}_{s t a b}(\varphi) \leq N-\frac{3}{2}$, then any iwip $\varphi$ is known not to be geometric, and in particular for any representative $\Phi_{k} \in \operatorname{Aut}\left(F_{N}\right)$ the fixed subgroup is trivial. The purpose of this paper is to show that the analogue of Masur and Smillie's result holds also in the non-maximal case:

Theorem 1.1. Let $N \geq 3$, and let $j_{1}, j_{2}, \ldots, j_{\ell}$ be any list of positive numbers from $\frac{1}{2} \mathbb{Z}$ which satisfy:

$$
\frac{1}{2} \leq \sum_{k=1}^{\ell} j_{k} \leq N-\frac{3}{2}
$$

Then there exists (and we give an explicit construction) an iwip automorphism $\varphi \in \operatorname{Out}\left(F_{N}\right)$ which realizes the given list of values $j_{k}$ as stable index list.

For $N=3$ the statement of the theorem had already been proved by C. Pfaff [11]. Other special cases were also known, for example the single 
element list $\left[N-\frac{3}{2}\right]$ for any $N \geq 3$ (see [6]). A further discussion, including some experimental data obtained by the first author, is given in section 7 below (compare also [4, Section VI]).

Remark 1.2. From Theorem 1.1 one deduces directly as corollary an analogous existence statement for indecomposable $\mathbb{R}$-trees $T$ with free isometric $F_{N}$-action that have prescribed branching index list given by the numbers $j_{k}$. This follows directly from the material assembled in Section 8 of 3 . The authors do not know whether such an existence statement was known previously.

Already in 4 the relationship between the index of $\varphi$ and the branching index of a forward limit $\mathbb{R}$-tree tree $T$ of $\varphi$ has been exploited (compare also [5]). If $\varphi$ is iwip, then such $T$ in the Thurston boundary $\partial \mathrm{cv}_{N}$ of (unprojectivized) Outer space $\mathrm{cv}_{N}$ is unique up to rescaling, and for non-geometric $\varphi$ the isometric $F_{N}$-action on $T$ is free and has dense orbits. For a suitable exponent $t \geq 1$ there is a natural 1-1 correspondence, between isogredience classes of representatives $\Phi_{k}$ of $\varphi^{t}$ with ind $\left(\Phi_{k}\right)>0$ on one hand, and $F_{N}$-orbits of branch points $P_{k}$ of $T$ on the other, where $2 \operatorname{ind}_{s t a b}\left(\Phi_{k}\right)+2$ is precisely equal to the number of directions at $P_{k}$. An exposition of this relationship is given in $\S 8$ of 3 .

This correspondence can be carried one step further by using the fact that $T$ is obtained as projective limit (in $\overline{\mathrm{cv}}_{N}=\mathrm{cv}_{N} \cup \partial \mathrm{cv}_{N}$ ) of simplicial metric trees $\widetilde{\Gamma}$ with free isometric $F_{N}$-action, which occur naturally as universal cover of a train track representative $f: \Gamma \rightarrow \Gamma$ of $\varphi$ (see \$2). Such train track representatives carry an intrinsic gate structure which allows one to define a gate index at every vertex of $\Gamma$ and a gate index list by considering all periodic vertices of $\Gamma$ with 3 or more gates. There is a natural relationship between the gates of $\widetilde{\Gamma}$ and the branching directions of $T$, which in the absence of so called periodic INPs (see \$2 below) becomes a 1-1 correspondence. Again, see $\S 8$ of [3] for more details.

The problem of realizing a given list $\left[j_{1}, j_{2}, \ldots, j_{\ell}\right]$ as in Theorem 1.1 as stable index list of an iwip automorphism can hence be subdivided into the following subproblems:

(1) Construct a graph $\Gamma$ with vertices $v_{1}, \ldots, v_{\ell}$ and define a gate structure $\mathbf{G}$ on $\Gamma$ which realizes the given list of the values $j_{k}$ as gate indices at the vertices $v_{k}$.

(2) Define a map $h: \Gamma \rightarrow \Gamma$ which respects the gate structure $\mathbf{G}$ and is "iwip up to INPs".

(3) Control the periodic INPs of $h$.

Subproblems (1) and (2) are solved below in sections 3 and 4. Subproblem (3), which is the hardest and conceptually the most interesting, requires a new tool, called long turns, which has been provided and investigated by the first two 
authors in the "companion paper" 3]. In section 5 we give a brief summary of this method and provide the concrete tools that allow us in section 6 to apply the results of [3] in order to obtain a legalizing train track morphism $g: \Gamma \rightarrow \Gamma$. It is then shown how Theorem 1.1 of [3] (quoted in section 6 in an appropriate version) can be applied to solve the left-over Subproblem (3) for the resulting train track map $h \circ g$.

Acknowledgements: This paper was intended by the authors to be joint with Catherine Pfaff: a large part of it is rooted in our weekly discussions with Catherine, during the months before she left Marseille. We regret that she declined despite our insistence to be coauthor of the paper.

We also would like to point the reader to the thesis work of Sonya Leibman [7, which came only very recently to our attention. Some of her results seem to be very interesting to the context of the work presented here; in particular, there is an overlap of the results of her section 5.2 (Lemma 5.4) and our subsection 7.1 below.

\section{Preliminaries}

We will use in this paper the same terminology as set up in sections 2 and 3 of [3]:

A graph $\Gamma$ is always connected, without vertices of valence 1 or 2 , and moreover it is finite, unless it is the universal covering of a finite connected graph. The edges $E^{ \pm}(\Gamma)$ of $\Gamma$ come in pairs $e, \bar{e}$ which differ only in their orientation, and $E^{+}(\Gamma)$ contains precisely one of the two elements in each pair.

A gate structure $\mathbf{G}$ on $\Gamma$ is a partition of the edges $e \in E^{ \pm}(\Gamma)$ into equivalence classes $\mathfrak{g}_{i}$ (called gates), where equivalent edges must have the same initial vertex $v$. Two edges $e, e^{\prime} \in E^{ \pm}(\Gamma)$ with same initial vertex form a turn $\left(e, e^{\prime}\right)$, which is called legal (with respect to $\mathbf{G}$ ) if $e$ and $e^{\prime}$ belong to distinct gates, and illegal if they belong to the same gate. The turn $\left(e, e^{\prime}\right)$ is called degenerate if $e=e^{\prime}$.

A path $\gamma=e_{1} e_{2} \ldots e_{q}$ crosses over a gate turn $\left(\mathfrak{g}_{i}, \mathfrak{g}_{j}\right)$ if for some $k \in$ $\{1, \ldots, q-1\}$ one has $\bar{e}_{k} \in \mathfrak{g}_{i}, e_{k+1} \in \mathfrak{g}_{j}$ or $\bar{e}_{k} \in \mathfrak{g}_{j}, e_{k+1} \in \mathfrak{g}_{i}$. The path $\gamma$ is legal if, for each $k \in\{1, \ldots, q-1\}$, the edges $\bar{e}_{k}$ and $e_{k+1}$ belong to different gates of $\mathbf{G}$ (i.e. $\gamma$ crosses only over legal turns).

The gate index $\operatorname{ind}_{\mathbf{G}}(v)$ at a vertex $v$ is given by $\operatorname{ind}_{\mathbf{G}}(v):=\frac{g(v)}{2}-1$, where $g(v)$ denotes the number of gates at $v$.

A graph map $f: \Gamma \rightarrow \Gamma$ maps vertices to vertices and edges to (possibly unreduced) edge paths. The map $f$ has no contracted edges if for any edge $e$ of $\Gamma$ the combinatorial length (= number of edges traversed) of $f(e)$ satisfies $|f(e)| \geq 1$. In this case $f$ induces a well defined map $D f$ on $E^{ \pm}(\Gamma)$ which maps the edge $e$ to the initial edge of the path $f(e)$. 
The transition matrix $M(f)=\left(m_{e^{\prime}, e}\right)_{e^{\prime}, e \in E^{+}(\Gamma)}$ of $f$ is defined as nonnegative matrix, where $m_{e^{\prime}, e}$ counts the number of times that $f(e)$ crosses over $e^{\prime}$ or over $\bar{e}^{\prime}$. The equality

$$
M(f \circ g)=M(f) M(g)
$$

is a direct consequence of the definition of the transition matrix. Recall that a non-negative matrix $M$ is called primitive if some positive power $M^{t}$ is positive, i.e. all coefficients of $M^{t}$ are strictly positive.

A graph map $f: \Gamma \rightarrow \Gamma$ is a train track morphism, with respect to a given gate structure $\mathbf{G}$ on $\Gamma$, if it has no contracted edges, and if $f$ maps every legal path to a legal path. It is shown in [3] that a train track morphism has the additional property that at every periodic vertex $v$ of $\Gamma$ any illegal turn is mapped to an illegal turn, or equivalently: $f$ induces at every periodic vertex $v$ of $\Gamma$ a bijective map from the gates at $v$ to the gates at $f(v)$. Note that in this paper all train track morphisms that occur have only periodic vertices; indeed, each vertex is a fixed point.

For a graph $\Gamma$ without preassigned gate structure, a train track map $f$ : $\Gamma \rightarrow \Gamma$ in the classical sense as defined by Bestvina and Handel [1] (and hence in particular any train track representative of a given automorphism of $F_{N}$ ) is a graph map with no contracted edges with the property that for any $t>0$ and any edge $e, f^{t}(e)$ is a reduced path.

As legal paths are reduced, any train track morphism $f: \Gamma \rightarrow \Gamma$ is always a classical train track map. Conversely, every classical train track map $f: \Gamma \rightarrow$ $\Gamma$ is a train track morphism with respect to the intrinsic gate structure $\mathbf{G}(f)$ on $\Gamma$, defined by $f$ through declaring two edges $e, e^{\prime}$ with same initial vertex to lie in the same gate if and only if for some $t \geq 1$ the edge paths $f^{t}(e)$ and $f^{t}\left(e^{\prime}\right)$ have non-trivial initial subpaths in common. Notice however that, for a train track morphism $f$ with respect to some gate structure $\mathbf{G}$, the intrinsic gate structure $\mathbf{G}(f)$ may be strictly finer than the given gate structure $\mathbf{G}$.

A reduced path $\gamma \circ \gamma^{\prime}$ in $\Gamma$ is a periodic INP for a train track morphism $f: \Gamma \rightarrow \Gamma$ if $\gamma$ and $\gamma^{\prime}$ are legal and for some $t \geq 1$ the path $f^{t}\left(\gamma \circ \gamma^{\prime}\right)$ is homotopic relative endpoints to $\gamma \circ \gamma^{\prime}$.

The gate-Whitehead-graph $W h_{\mathbf{G}}^{v}(f)$ of a train track morphism $f: \Gamma \rightarrow \Gamma$ at a vertex $v$ of $\Gamma$ has the gates $\mathfrak{g}_{i}$ of $\mathbf{G}$ at $v$ as vertices and a (non-oriented) edge connecting $\mathfrak{g}_{i}$ to $\mathfrak{g}_{j}$ if and only for some edge $e$ of $\Gamma$ and some integer $t \geq 1$ the edge path $f^{t}(e)$ crosses over the gate turn $\left(\mathfrak{g}_{i}, \mathfrak{g}_{j}\right)$.

Recall also that an automorphism $\varphi \in \operatorname{Out}\left(F_{N}\right)$ is called iwip (or fully irreducible) if no positive power of $\varphi$ fixes the conjugacy class of any proper free factor of $F_{N}$.

\section{The GRAPH $\Gamma$ WITH PRESCRIBED INDEX LIST}

In this and the following sections, let $N, \ell$ and $j_{1}, \ldots, j_{\ell}$ be given as in Theorem 1.1. In this section we will build a graph $\Gamma$ with $\pi_{1} \Gamma \cong F_{N}$ which 
has precisely $\ell$ vertices $v_{1}, \ldots, v_{\ell}$, and has at each vertex $v_{k}$ precisely $i_{k}:=$ $2 j_{k}+2 \geq 3$ gates: the gate structure $\mathbf{G}$ on $\Gamma$ realizes the given list $j_{1}, \ldots, j_{\ell}$ as gate index list.

Note that from the inequalities in Theorem 1.1 we obtain

$$
1 \leq i_{1}+\cdots+i_{\ell}-2 \ell \leq 2 N-3
$$

as initial assumption on the number of gates in $\Gamma$.

We divide the possible index lists in three different cases:

(1) The even case: $i_{1}+\cdots+i_{\ell}$ is even (that is to say $j_{1}+\cdots+j_{\ell}$ is an integer $\leq N-2)$.

(2) The odd case (non-maximal): $i_{1}+\cdots+i_{\ell}$ is odd and smaller than $2 N-4+2 \ell$ (alternatively: $j_{1}+\cdots+j_{\ell} \leq N-2$ ).

(3) The maximal odd case: $i_{1}+\cdots+i_{\ell}=2 N-3+2 \ell$ (i.e. $j_{1}+\cdots+j_{\ell}=$ $\left.N-\frac{3}{2}\right)$.

We consider a circle which is subdivided at vertices labeled $v_{1}, \ldots, v_{\ell}$, to obtain oriented edges labeled $c_{1}, \ldots, c_{\ell}$ such that $c_{k}$ starts at $v_{k}$ and ends at $v_{k+1}$ (for $k$ understood modulo $\ell$ ). Note that if $\ell=1$ then $c_{1}=c_{\ell}$ is a loop edge at the sole vertex $v_{1}$ of $\Gamma$.

At each vertex $v_{k}$ we add $i_{k}-2$ germs of edges to this circle. In the odd and maximal odd cases we remove one of these germs at $v_{1}$ such that in any cases the number of germs is even. We group these germs arbitrarily into pairs to form $r$ oriented edges $b_{1}, \ldots, b_{r}$. Here $r$ is the largest integer $\leq j_{1}+\cdots+j_{\ell}$, with $r=0$ exactly if the index list is equal to $\left[\frac{1}{2}\right]$.

In the even and odd cases let $s=N-r-1$, where we note that $s \geq 1$. We add $s$ oriented edges $a_{1}, \ldots, a_{s}$ which are loops at the vertex $v_{1}$.

In the maximal odd case we set $s=1$ and add a single edge $a_{1}$ which is a loop at $v_{1}$.

Finally, in the odd case we add an extra edge $d$ which is a loop at $v_{1}$.

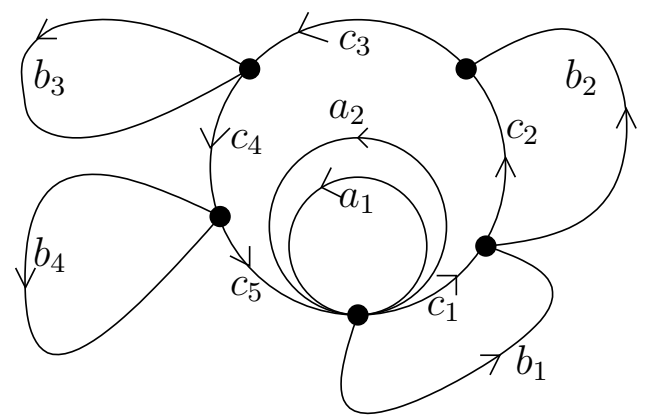

Figure 1. Even case: a graph $\Gamma$ with index list $\left[\frac{1}{2}, 1, \frac{1}{2}, 1,1\right]$ and $N=7$ 


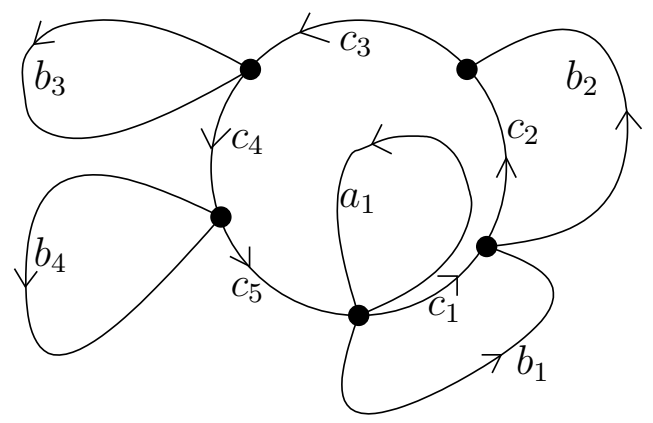

Figure 2. Maximal odd case: a graph $\Gamma$ with index list $\left[1,1, \frac{1}{2}, 1,1\right]$ and $N=6$

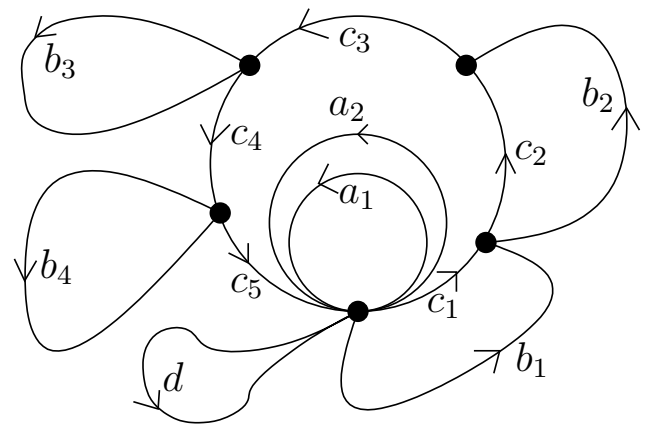

Figure 3. Odd case: a graph $\Gamma$ with index list $\left[1,1, \frac{1}{2}, 1,1\right]$ and $N=8$

The graphs $\Gamma$ defined above are connected, without vertices of valence 1 or 2, and have fundamental group $F_{N}$. The oriented edge set is given by $E^{+}(\Gamma)=\left\{c_{1}, \ldots, c_{\ell}, b_{1}, \ldots, b_{r}, a_{1}, \ldots a_{s}\right\}$ in the even and maximal odd cases, and by $E^{+}(\Gamma)=\left\{c_{1}, \ldots, c_{\ell}, b_{1}, \ldots, b_{r}, a_{1}, \ldots a_{s}, d\right\}$ in the odd case. In all cases we have $\ell, s \geq 1$ and $r \geq 0$, with $r=0$ if and only if we are in the odd case with index list $\left[\frac{1}{2}\right]$.

We define the gate structure $\mathbf{G}$ on $\Gamma$ in such a way that each gate consists of a single edge, except for the following gates, all based at $v_{1}$ :

- in the even case: $\mathfrak{g}_{1}=\left\{c_{1}, a_{1}, \ldots a_{s}\right\}, \mathfrak{g}_{2}=\left\{\bar{c}_{\ell}, \bar{a}_{1}, \ldots, \bar{a}_{s}\right\}$;

- in the odd case: $\mathfrak{g}_{1}=\left\{c_{1}, a_{1}, \ldots, a_{s}\right\}, \mathfrak{g}_{2}=\left\{\bar{c}_{\ell}, \bar{a}_{1}, \ldots, \bar{a}_{s}\right\}$ and $\mathfrak{g}_{3}=$ $\{d, \bar{d}\}$

- in the maximal odd case: $\mathfrak{g}_{1}=\left\{c_{1}, a_{1}\right\}$.

Notice that in the maximal odd case $\bar{a}_{1}$ and $\bar{c}_{\ell}$ belong to distinct gates. 
As a consequence, at every vertex $v_{k}$ there are precisely $i_{k}$ gates, so that we obtain:

Proposition 3.1. The gate structure $\mathbf{G}$ on $\Gamma$ realizes the given list of values $j_{1}, \ldots, j_{\ell}$ as gate indices at the vertices $v_{1}, \ldots, v_{\ell}$ of $\Gamma$.

The following will be used crucially in the subsequent sections:

Lemma 3.2. Let $\Gamma$ be the graph equipped with the gate structure $\mathbf{G}$ as defined above.

(1) For each edge $e \neq a_{1}$ in $E^{+}(\Gamma)$ there exists a legal loop ueu' in $\Gamma$ which starts in $\mathfrak{g}_{1}$ does not end in $\mathfrak{g}_{1}$, does not pass through $a_{1}, \bar{a}_{1}$ or $\bar{e}$ and passes exactly once through e (we allow $u$ or $u^{\prime}$ to be trivial).

(2) For each gate turn $t=\left(\mathfrak{g}, \mathfrak{g}^{\prime}\right)$, except for gate turns involving $\left\{\bar{a}_{1}\right\}$ in the maximal odd case, there exists a legal loop $v$ which starts in $\mathfrak{g}_{1}$, does not end in $\mathfrak{g}_{1}$, does not pass through $a_{1}$ or $\bar{a}_{1}$, and crosses over the gate turn $\left(\mathfrak{g}, \mathfrak{g}^{\prime}\right)$.

(3) In the even and odd cases, for any edge $e$ in $\mathfrak{g}_{1}$ there exist legal paths $\alpha$ and $\beta$ that do not pass through any of the $a_{i}$ or through $e$ (and

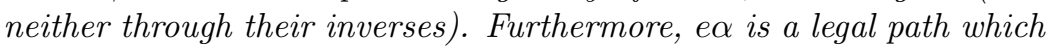
ends in $\mathfrak{g}_{2}$, and $\beta$ is a legal loop based at $v_{1}$ that does not start in $\mathfrak{g}_{1}$ or $\mathfrak{g}_{2}$, and does not end in $\mathfrak{g}_{1}$. We allow $\alpha$ to be trivial.

(4) Symmetrically, in the even and odd cases, for each edge $\bar{e}$ in $\mathfrak{g}_{2}$ there exist legal paths $\alpha^{\prime}$ and $\beta^{\prime}$ that do not pass through any of the $a_{i}$ or through e (nor through their inverses), such that $\alpha^{\prime} e$ is legal and starts in $\mathfrak{g}_{1}$, while the legal loop $\beta^{\prime}$ is based at $v_{1}$ but does not start in $\mathfrak{g}_{2}$ and does not end in $\mathfrak{g}_{1}$ or $\mathfrak{g}_{2}$. We allow $\alpha^{\prime}$ to be trivial.

Proof. The above statements (1) and (2) are easy to verify if one keeps in mind that at every vertex there are $\geq 3$ gates, and that every vertex $v_{k}$ can be reached from $v_{1}$ by any one of two disjoint paths on the circle $c_{1} \cdots c_{\ell}$, so that it is easy to avoid any given edge in $\Gamma$.

Concerning statement (3), if $e \neq c_{1}$ or $\ell=1$, we let $\alpha$ be trivial. Otherwise we set $\alpha=c_{2} \cdots c_{\ell}$. In the odd case we let $\beta=d$. In the even case, there is at least one edge $b_{k}$ (or $\bar{b}_{k}$ ) exiting from $v_{1}$. Let $v_{k^{\prime}}$ be the endpoint of $b_{k}$ (or of $\left.\bar{b}_{k}\right)$, and set $\beta=b_{k}$ if $k^{\prime}=1$ and $\beta=b_{k} c_{k^{\prime}} \cdots c_{\ell}$ otherwise.

For statement (4) the paths $\alpha^{\prime}$ and $\beta^{\prime}$ can be chosen symmetrically to $\alpha$ and $\beta$ in the above case (3).

\section{THE TRAIN TRACK MORPHISM}

In this section we construct for the graph $\Gamma$ a train track morphism $h$ : $\Gamma \rightarrow \Gamma$ with respect to the gate structure $\mathbf{G}$ specified in the last section. The morphism $h$ will be "fully irreducible up to INPs" in that it has primitive transition matrix $M(h)$ and connected gate-Whitehead-graphs at every vertex (compare [3, Propositions 4.1 and 4.2]). 
Below we will consider graph maps $f: \Gamma \rightarrow \Gamma$ with the following properties:

(1) $f$ is a homotopy equivalence,

(2) $f$ is a train track morphism with respect to the gate structure $\mathbf{G}$,

(*) (3) $f$ fixes each vertex of $\Gamma$,

(4) $f$ fixes each gate of $\mathbf{G}$, and

(5) the $f$-image of every edge $e$ crosses over $e$.

Lemma 4.1. Let $f_{1}$ and $f_{2}$ be graph maps which satisfy the above Properties $(*)$.

(a) Then the composition $f_{1} \circ f_{2}$ satisfies $(*)$ as well.

(b) Moreover, for any vertex $v_{k}$ of $\Gamma$ the gate-Whitehead-graph $W h_{\mathbf{G}}^{v_{k}}\left(f_{1} \circ\right.$ $\left.f_{2}\right)$ contains both gate-Whitehead-graphs $W h_{\mathbf{G}}^{v_{k}}\left(f_{1}\right)$ and $W h_{\mathbf{G}}^{v_{k}}\left(f_{2}\right)$ as subgraphs.

Proof. Statement (a) is a direct consequence of the definition of properties (*). Statement (b) has been proved under slightly more general hypotheses as Proposition 3.10 in [3].

The Properties (3) and (4) above imply that a map which satisfies $(*)$ acts as identity on the set of gate turns. As a consequence one derives easily that Statement $(\mathrm{b})$ of Lemma 4.1 can actually improved to $W h_{\mathbf{G}}^{v_{k}}\left(f_{1} \circ f_{2}\right)=$ $W h_{\mathbf{G}}^{v_{k}}\left(f_{1}\right) \cup W h_{\mathbf{G}}^{v_{k}}\left(f_{2}\right)$.

We define below several graph maps on $\Gamma$ where we use the following:

Convention 4.2. In this and the following sections, in the definition of a graph map $\Gamma \rightarrow \Gamma$ we always use the convention that any edge with no explicitly defined image is mapped identically to itself.

For any edge $e \neq a_{1} \in E^{+}(\Gamma)$ let $u$ and $u^{\prime}$ be as in Lemma 3.2 (1). Define $h_{e}: \Gamma \rightarrow \Gamma$ by:

$$
\begin{aligned}
h_{e}: a_{1} & \mapsto u e u^{\prime} a_{1} \\
e & \mapsto e u^{\prime} a_{1} u e
\end{aligned}
$$

Note that the $h_{e}$-image of $e$ passes through $a_{1}$ and that the $h_{e}$ image of $a_{1}$ passes through $e$.

For any gate turn $t=\left(\mathfrak{g}, \mathfrak{g}^{\prime}\right)$ of $\Gamma$, except for gate turns involving $\left\{\bar{a}_{1}\right\}$ in the maximal odd case, let $v$ be as in Lemma 3.2 (2) and define $h_{t}: \Gamma \rightarrow \Gamma$ by:

$$
h_{t}: a_{1} \mapsto v a_{1}
$$

Let $h^{\prime}$ be the composition of all these maps $h_{e}$ (with $e \neq a_{1}$ ) and $h_{t}$, where we do not care about the order of the composition. Define $h: \Gamma \rightarrow \Gamma$ through $h=h^{\prime} \circ h^{\prime}$.

Proposition 4.3. The map $h: \Gamma \rightarrow \Gamma$ is a train track morphism with respect to $\mathbf{G}$. Furthermore $h$ fixes every vertex $v_{k}$ of $\Gamma$, maps each gate of the gate structure $\mathbf{G}$ to itself, and is a homotopy equivalence. 
In addition, the transition matrix $M(h)$ is positive, and the gate-Whiteheadgraph $W h_{\mathbf{G}}^{v}(h)$ of $h$ at any vertex $v$ of $\Gamma$ is connected.

Proof. We first consider the maps $h_{e}$ with $e \neq a_{1}$ and $h_{t}$ as defined above. Properties (2)-(5) of $(*)$ above are easily verified directly. For Property (1) the reader can check directly that the map given by

$$
\begin{aligned}
a_{1} & \mapsto \bar{u}^{\prime} \bar{e} \bar{u} a_{1}^{2} \\
e & \mapsto \bar{u} \bar{a}_{1} u e
\end{aligned}
$$

is a homotopy inverse of $h_{e}$. The fact that it is not a train track map with respect to $\mathbf{G}$ is irrelevant. For $h_{t}$ a homotopy inverse is given simply by $a_{1} \mapsto \bar{v} a_{1}$. From Lemma 4.1 it follows now that the maps $h^{\prime}$ and $h$ have this Property $(*)$, which is the statement of the first paragraph to be shown.

In order to show that $M(h)$ is positive, we use the equality $M(f g)=$ $M(f) M(g)$ from Section 2 and condition (5) of Property $(*)$ to obtain that the image $h^{\prime}(e)$ of any edge $e$ crosses over $a_{1}$, and that the image $h^{\prime}\left(a_{1}\right)$ of $a_{1}$ crosses over every edge $e \in E^{+}(\Gamma)$. Hence the image $h(e)$ of any edge $e$ passes through all edges of $\Gamma$. This proves that the transition matrix $M(h)$ is positive.

From Lemma 4.1 we know that the gate-Whitehead-graph of $h$ contains that of $h_{t}$, for each gate turn $t=\left(\mathfrak{g}, \mathfrak{g}^{\prime}\right)$. It follows from the above definition of $h_{t}$ via $a_{1} \mapsto v a_{1}$ and from the definition of $v$ in Lemma 3.2 (2) that in the even and the odd cases the gate-Whitehead-graph of $h$ at each vertex of $\Gamma$ is a complete graph and thus connected. In the maximal odd case there are no maps $h_{t}$ for the gate turns involving the gate $\left\{\bar{a}_{1}\right\}$. But in this case the gate turn $\left(\left\{\bar{a}_{1}\right\},\left\{a_{1}, c_{1}\right\}\right)$ is crossed over by $h_{c_{1}}\left(c_{1}\right)$. This is enough to get that the gate-Whitehead-graph of $h$ at $v_{1}$ is connected.

\section{BuILDing THE LEGALIZING MAP}

The goal of this (and the following) section is to construct a train track morphism $g: \Gamma \rightarrow \Gamma$ with respect to $\mathbf{G}$ which is a homotopy equivalence and is "legalizing". This notion has been introduced in [3], and is now briefly summarized:

A pair $\left(\gamma, \gamma^{\prime}\right)$ of non-trivial legal paths $\gamma$ and $\gamma^{\prime}$ in $\Gamma$ is called in 3 a long turn if the branches $\gamma$ and $\gamma^{\prime}$ start at the same vertex but have distinct initial edges. The set of long turns in $\Gamma$, with both branches of length equal to some integer $C \geq 1$, is denoted by $L T_{C}(\Gamma)$.

The long turn $\left(\gamma, \gamma^{\prime}\right)$ can be legal or illegal, according to whether its starting turn $s\left(\gamma, \gamma^{\prime}\right)$, formed by the initial edges of $\gamma$ and $\gamma^{\prime}$ respectively, is legal or illegal (as defined for turns in the traditional sense, see \$2).

If neither $g(\gamma)$ is a subpath of $g\left(\gamma^{\prime}\right)$ nor conversely, then the long turn is called $g$-long, and the long turn, obtained from $g(\gamma)$ and $g\left(\gamma^{\prime}\right)$ through erasing from both the maximal common initial subpath, is called the $g$-image of $\left(\gamma, \gamma^{\prime}\right)$ 
and denoted by $g^{L T}\left(\gamma, \gamma^{\prime}\right)$. A train track morphism $g$ is called legalizing if for some sufficiently large constant $C \geq 1$ every long turn $\left(\gamma, \gamma^{\prime}\right) \in L T_{C}(\Gamma)$ is $g$-long, and if $g^{L T}\left(\gamma, \gamma^{\prime}\right)$ is legal (or, equivalenty, if the starting turn of $g^{L T}\left(\gamma, \gamma^{\prime}\right)$ is legal $)$.

To avoid a misunderstanding, we point out that any non-degenerate turn $\left(e, e^{\prime}\right)$ in the classical sense can be considered alternatively as long turn with branches of length 1. In particular, if $\left(e, e^{\prime}\right)$ is $g$-long, then it has both, an image turn $\left(D g(e), D g\left(e^{\prime}\right)\right)$, as well as an image long turn $g^{L T}\left(e, e^{\prime}\right)$, which furthermore has a starting turn $s g^{L T}\left(e, e^{\prime}\right)$ that is again a turn in the classical sense. However, in general $\left(D g(e), D g\left(e^{\prime}\right)\right)$ and $s g^{L T}\left(e, e^{\prime}\right)$ will be quite different: for example, $\left(D g(e), D g\left(e^{\prime}\right)\right)$ may well be degenerate, while $s g^{L T}\left(e, e^{\prime}\right)$ is by definition always non-degenerate.

To construct the desired train track morphism $g$ we define now train track morphisms $g_{t}$. In each of the cases considered below the "variable" $t$ denotes a non-degenerate illegal turn in $\Gamma$, interpreted here as long turn with branch length 1 . The reader can verify directly that all of the maps $g_{t}$ defined below satisfy the statements (2), (3) and (4) of Property $(*)$ from Section 4. We use again Convention 4.2 .

We first deal with the even and odd cases:

(1) Let $t=\left(a_{i}, e\right)$ be an illegal turn in $\Gamma$ with $1 \leq i \leq s$, and with $e=c_{1}$ or $e=a_{j}$ where $i \neq j$. Let $\alpha$ and $\beta$ be as in Lemma 3.2. Define:

$$
\begin{aligned}
g_{t}: a_{i} & \mapsto a_{i} e \alpha \\
e & \mapsto a_{i} \beta a_{i} e
\end{aligned}
$$

The illegal turn $t=\left(a_{i}, e\right)$ is $g_{t}$-long and mapped by $g_{t}^{L T}$ to the long turn $\left(e \alpha, \beta a_{i} e\right)$ which is legal.

(2) Symmetrically, let $t=\left(\bar{a}_{i}, \bar{e}\right)$ be an illegal turn in $\Gamma$ with $1 \leq i \leq s$, and with $e=c_{\ell}$ or $e=a_{j}$ where $i \neq j$. Let $\alpha^{\prime}$ and $\beta^{\prime}$ be as in Lemma 3.2, Define:

$$
\begin{aligned}
g_{t}: a_{i} & \mapsto \alpha^{\prime} e a_{i} \\
e & \mapsto e a_{i} \beta^{\prime} a_{i}
\end{aligned}
$$

The illegal turn $t=\left(\bar{a}_{i}, \bar{e}\right)$ is $g_{t}$-long and mapped by $g_{t}^{L T}$ to the long turn $\left(\bar{e} \bar{\alpha}^{\prime}, \bar{\beta}^{\prime} \bar{a}_{i} \bar{e}\right)$ which is legal.

(3) In the odd case we have one more illegal turn $t=(d, \bar{d})$. Define:

$$
\begin{aligned}
g_{t}: a_{1} & \mapsto a_{1} \bar{d} c_{1} \cdots c_{\ell} \\
d & \mapsto d a_{1} \bar{d}
\end{aligned}
$$

The illegal turn $t=(d, \bar{d})$ is $g_{t}$-long and mapped by $g_{t}^{L T}$ to the long turn $\left(a_{1} \bar{d}, \bar{a}_{1} \bar{d}\right)$ which is legal.

In the maximal odd case there is only one illegal turn $t=\left(a_{1}, c_{1}\right)$. As the rank $N$ is greater or equal to 3 , there is at least one edge $b_{1}$ which starts from 
some vertex $v_{k}$ and ends at some $v_{k^{\prime}}$. We set $c_{[1, k]}=c_{1} \cdots c_{\ell}$ if $k=1$ and $c_{[1, k]}=c_{1} \cdots c_{k-1}$ if $2 \leq k \leq \ell$. We furthermore set $c_{\left[k^{\prime}, \ell\right]}=1$ if $k^{\prime}=1$, and $c_{\left[k^{\prime}, \ell\right]}=c_{k^{\prime}} \cdots c_{\ell}$ if $2 \leq k^{\prime} \leq \ell$.

We define:

$$
\begin{aligned}
g_{t}: & a_{1} \\
c_{1} & \mapsto c_{1} \cdots c_{\ell} \cdots c_{\ell} c_{[1, k]} b_{11, k]} c_{\left[k_{1}, \ell\right]} c_{\left[k^{\prime}, \ell\right]} a_{1} c_{1} \\
b_{1} & \mapsto b_{1} c_{\left[k^{\prime}, \ell\right]} a_{1} c_{[1, k]} b_{1}
\end{aligned}
$$

Lemma 5.1. In the maximal odd case, every long turn of length equal to $\ell+1$ with starting turn $\left(a_{1}, c_{1}\right)$ is $g_{t}$-long and mapped by $g_{t}^{L T}$ to a legal long turn.

Proof. Let $t^{*}=\left(a_{1} e_{2} \ldots e_{\ell+1}, c_{1} e_{2}^{\prime} \ldots e_{\ell+1}^{\prime}\right)$ be the long turn under consideration. We first observe that, if $e_{2} \neq c_{1}$ and $e_{2} \neq a_{1} \rrbracket$, then $g_{t}^{L T}\left(t^{*}\right)$ has starting turn $\left(e_{2}, c_{1}\right)$ and hence is legal. In order to treat computationally the possible "exceptional" cases without too many subcases we introduce a variable $x$ which we set to $x=a_{1} c_{1}$ if $e_{2}=c_{1}$ and $x=a_{1}$ if $e_{2}=a_{1}$. Similarly, a second variable $y$ will be used below which is set to $y=a_{1} c_{1}$ if $e_{\ell+1}^{\prime}=c_{1}$ and $y=a_{1}$ if $e_{\ell+1}^{\prime}=a_{1}$.

We observe that in each case $g_{t}^{L T}\left(t^{*}\right)$ is legal unless $e_{2}^{\prime} \ldots e_{\ell+1}^{\prime}=c_{2} \ldots c_{\ell} c_{1}$ or $e_{2}^{\prime} \ldots e_{\ell+1}^{\prime}=c_{2} \ldots c_{\ell} a_{1}$. We compute

$$
g_{t}^{L T}\left(t^{*}\right)=\left(b_{1} c_{\left[k^{\prime}, \ell\right]} x, c_{k} \cdots c_{\ell} c_{[1, k]} b_{1} c_{\left[k^{\prime}, \ell\right]} y\right) \text { if } \ell \geq k \geq 2,
$$

and

$$
g_{t}^{L T}\left(t^{*}\right)=\left(b_{1} c_{\left[k^{\prime}, \ell\right]} x, c_{1} \ldots c_{\ell} b_{1} c_{\left[k^{\prime}, \ell\right]} y\right) \text { if } k=1 \text { and } \ell \geq 2 .
$$

Finally we have

$$
g_{t}^{L T}\left(t^{*}\right)=\left(b_{1} c_{\left[k^{\prime}, \ell\right]} x, c_{1} b_{1} c_{\left[k^{\prime}, \ell\right]} y\right) \text { if } k=\ell=1,
$$

All three of those computed long turns are legal.

We now verify:

Lemma 5.2. Each of the above defined maps $g_{t}$ is a homotopy equivalence.

Proof. For each case of the map $g_{t}$ we list below a map $g_{t}^{\prime}$; the reader can verify directly that they are homotopy inverses of the maps $g_{t}$.

Even and odd cases:

$$
\begin{aligned}
g_{t}^{\prime}: a_{i} & \mapsto e \alpha \bar{a}_{i} \bar{\beta} \\
e & \mapsto \beta a_{i} \bar{\alpha} \bar{e} a_{i} \bar{\alpha} \\
g_{t}^{\prime}: a_{i} & \mapsto \bar{\beta}^{\prime} \bar{a}_{i} \alpha^{\prime} e \\
e & \mapsto \bar{\alpha}^{\prime} a_{i} \bar{e} \bar{\alpha}^{\prime} a_{i} \beta^{\prime}
\end{aligned}
$$

1 We'd like to thank C. Pfaff for having pointed out to us that the treatment of this case was missing in an earlier version of our paper. 
(3)

$$
\begin{aligned}
g_{t}^{\prime}: a_{1} & \mapsto a_{1} \bar{c}_{\ell} \ldots \bar{c}_{1} d c_{1} \ldots c_{\ell} \bar{a}_{1} \\
d & \mapsto d c_{1} \ldots c_{\ell} \bar{a}_{1}
\end{aligned}
$$

Maximal odd case:

$$
\begin{aligned}
g_{t}^{\prime}: a_{1} & \mapsto \bar{c}_{\left[k^{\prime}, \ell\right]} \bar{b}_{1} \bar{c}_{[1, k]} a_{1} \bar{c}_{\ell} \ldots \bar{c}_{1} a_{1}^{2} \bar{c}_{\ell} \ldots \bar{c}_{1} a_{1}^{2} \\
c_{1} & \mapsto \bar{a}_{1} c_{1} \\
b_{1} & \mapsto \bar{c}_{[1, k]} \bar{a}_{1} c_{1} \ldots c_{\ell} \bar{a}_{1} c_{[1, k]} b_{1}
\end{aligned}
$$

We thus have proved:

Proposition 5.3. Let $L=1$ in the even and odd cases and $L=\ell+1$ in the maximal odd case, and let $t$ be any illegal turn of $\Gamma$. For each long turn $t^{*}$ of $\Gamma$, with branch length $L$ and with starting turn $t$, the map $g_{t}$ is a train track morphism with the property that $t^{*}$ is $g_{t}$-long and mapped by $g_{t}^{L T}$ to a legal long turn.

Furthermore, $g_{t}$ is a homotopy equivalence which fixes every vertex of $\Gamma$ and every gate of $\mathbf{G}$.

\section{Proof of the main Result}

Proposition 5.3 is the main ingredient we need to obtain the desired legalizing map. This is done through an application of Proposition 7.1 of [3] which we quote now, for the convenience of the reader in a slightly weakened form and with terminology adapted to the present paper:

Proposition 6.1 ([3, Proposition 7.1]). Let $\Gamma$ be a graph with a gate structure G. Assume that there exists a constant $L \geq 1$, and assume furthermore:

(1) For each illegal long turn $t$ with branch length $L$ there exists a train track morphism $g_{t}: \Gamma \rightarrow \Gamma$ such that $t$ is $g_{t}$-long and mapped by $g_{t}^{L T}$ to a legal long turn.

(2) There exists a train track morphism $h: \Gamma \rightarrow \Gamma$ which satisfies $|h(e)| \geq$ 2 for any edge e of $\Gamma$.

(3) All the above maps $g_{t}$ and $h$ are homotopy equivalences.

Then there exists a legalizing train track morphism $g: \Gamma \rightarrow \Gamma$ which is obtained as a composition of the $g_{t}$ and $h$.

Before going back to the situation considered in the previous sections, we first quote the main result of [3], in a slightly strengthened version due to Remark 6.6 of 3 and adapted to the circumstances here:

Theorem 6.2 ([3, Theorem 1.1 and Remark 6.6]). Let $\Gamma$ be a graph with gate structure $\mathbf{G}$, let $f: \Gamma \rightarrow \Gamma$ be a train track morphism with positive transition matrix $M(f)$ and gate-Whitehead-graph at every vertex that is connected. Let $g: \Gamma \rightarrow \Gamma$ be a legalizing train track morphism with respect to the gate structure 
$\mathbf{G}$ which is a homotopy equivalence that fixes every vertex of $\Gamma$ and every gate of $\mathbf{G}$. Then:

(1) The map $f \circ g: \Gamma \rightarrow \Gamma$ is a train track representative of an iwip automorphism $\varphi \in \operatorname{Out}\left(F_{N}\right)$.

(2) There is no periodic INP in $\Gamma$ for the train track map $f \circ g$. In particular there are no non-trivial $(f \circ g)$-periodic conjugacy classes in $F_{N}$.

(3) The stable index list for $\varphi$ is given by the gate index list for $\Gamma$ defined by $\mathbf{G}$ at the $f$-periodic vertices of $\Gamma$.

We will now go back to the graph $\Gamma$ from the previous sections, i.e. with gate structure $\mathbf{G}$ that realizes the given index list from Theorem 1.1 as gate indices. We will show below how to use the previously derived train track morphisms $h$ and $g_{t}$ on $\Gamma$ via the above quoted results from [3] to finish the proof of Theorem 1.1 .

We first observe:

Corollary 6.3. Let $\Gamma$ be the graph with gate structure $\mathbf{G}$ defined in Section 3 for the given list of gate indices. Then there exists a legalizing train track morphism $g: \Gamma \rightarrow \Gamma$ which is a homotopy equivalence and fixes each vertex and each gate of $\Gamma$.

Proof. We use Proposition 5.3 to obtain the hypothesis (1) of Proposition 6.1. where we note that if $g_{t}$ legalizes a long turn $t^{\prime}$ with branch length $C^{\prime} \geq 1$, then it also legalizes any long turn $t$ with branch length $C \geq C^{\prime}$ which contains $t^{\prime}$ as "subturn".

We note that hypothesis (2) of Proposition 6.1 is satisfied by the train track morphism $h: \Gamma \rightarrow \Gamma$ from Proposition 4.3 .

Hypothesis (3) is satisfied, as has been shown in Propositions 4.3 and 5.3

Thus Proposition 6.1 assures us the existence of the legalizing map $g$ as product of $h$ and the $g_{t}$. Since by Propositions 4.3 and 5.3 the latter are all homotopy equivalences that fix every vertex of $\Gamma$ and every gate of $\mathbf{G}$, this proves the claim.

Proof of Theorem 1.1. We note that the map $h: \Gamma \rightarrow \Gamma$ from Section 4 satisfies by Proposition 4.3 all of the requirements of the map $f$ in Theorem 6.2. By Corollary 6.3 the same is true for the map $g$ obtained in Corollary 6.3.

Hence part (11) of Theorem 6.2 shows that $h \circ g$ represents an iwip automorphism $\varphi$ of $F_{N}=\pi_{1} \Gamma$, and part (3) assures that the stable index list of $\varphi$ is equal to the gate index list of $\Gamma$ with respect to $\mathbf{G}$, which is built in Section 3 to realize the given list of values $j_{1}, j_{2}, \ldots, j_{\ell}$, see Proposition 3.1

Remark 6.4. There is a subtlety in the above proof which we would like to point out to the reader, concerning the topic "given gate structure" versus "intrinsic gate structure" (as defined in Section 2). It shows up in relation to two aspects which are relevant in our context: 
(a) The index of the automorphism represented by a train track morphisms $f: \Gamma \rightarrow \Gamma$ with respect to a gate structure $\mathbf{G}$ depends on the intrinsic gate structure $\mathbf{G}(f)$, which may well be strictly finer than G.

(b) A map $g: \Gamma \rightarrow \Gamma$, which is a train track morphism with respect to two distinct gate structures $\mathbf{G}$ and $\mathbf{G}^{\prime}$, may well be legalizing with respect to $\mathbf{G}$ but not with respect $\mathbf{G}^{\prime}$. (In this case, however, $\mathbf{G}$ must be strictly finer than $\mathbf{G}^{\prime}$ ).

In the situation considered above, both potential problems are resolved as follows by the application of Theorem 6.2 .

The train track morphism $h$ constructed in Section 4 may indeed well have an intrinsic gate structure $\mathbf{G}(h)$ that is strictly finer than the previously defined gate structure G. However, in Lemma 5.9 of [3] it has been shown that a train track morphisms $g$ with respect to a gate structure $\mathbf{G}$ which is legalizing (with respect to $\mathbf{G}$ ) satisfies indeed $\mathbf{G}(g)=\mathbf{G}$. Now, since the composition of any train track morphism with a legalizing train track morphism is again legalizing (see [3, Proposition 5.8]), by the same argument as before we obtain automatically $\mathbf{G}(h \circ g)=\mathbf{G}$.

\section{DisCUSSION}

We will now discuss some further aspects of the index of free group automorphisms:

7.1. The index deficit. Handel and Mosher [5, §1.5, Question 5] ask what values for the index deficit $N-\operatorname{ind}_{\text {stab }} \varphi-1$ for any (iwip) automorphism $\varphi \in \operatorname{Out}\left(F_{N}\right)$ are possible, and whether for $N \rightarrow \infty$ the maximal index deficit goes to $\infty$.

From our Theorem 1.1 it follows directly that for every $N \geq 3$ every value $j$ in $\frac{1}{2} \mathbb{N}$ with $0 \leq j \leq N-\frac{3}{2}$ is achieved as index deficit for some iwip $\varphi$. The maximal index deficit is hence equal to $N-\frac{3}{2}$, which indeed tends to $\infty$ for $N \rightarrow \infty$. This result has independently been obtained also by Sonya Leibman [7.

7.2. The index of geometric iwips. We now consider in some detail the results of Masur and Smillie [10], in particular their Theorem 2 on p. 291: The translation of the terminology used there for quadratic differentials and pseudo-Anosov homeomorphisms to the usual terminology for free group automorphisms is not completely evident. We give here a bit of translation help:

In the absence of punctures on the surface $M$, a $p_{k}$-pronged singularity in [10] translates into an isogredience class of automorphisms $\Phi_{k}$ with ind $\Phi_{k}=$ $j_{k}=\frac{p_{k}-2}{2}$. In this case we would have to translate the genus $g$ of $M$, multiplied by 2 , into the rank $N$ of the free group, except that without punctures $\pi_{1} M$ 
is not free, which explains the summand -4 in the index equality in part (a) of Theorem 2 of [10].

Now, the $n$ punctures which Masur and Smillie admit in their Theorem 2 appear nowhere explicitly, but in fact they can be essentially anywhere: If a puncture lies outside of the singularities and outside the separatrices raying out from them, then it lies on some regular leaf of the stable foliation, and hence it becomes a "2-prong singularity", thus contributing a value $p_{k}=2$ to the given list. The automorphisms $\Phi_{k}$ in the corresponding isogredience class have two attracting fixed points on $\partial F_{N}$ and $\operatorname{rank}\left(\operatorname{Fix} \Phi_{k}\right)=1$, which adds up to ind $\Phi_{k}=1$.

If a puncture coincides with a singularity, say with $p_{k}$ prongs, then, by the analogous reasoning, we obtain ind $\Phi_{k}=\frac{p_{k}}{2}$. This explains also why a value $p_{k}=1$, which they admit, does not lead to negative index of the corresponding isogredience class: any singularity with a single prong only must coincide with one of the punctures!

However, in the context of the paper here we have to add a further restriction: A pseudo-Anosov surface homeomorphism induces an iwip automorphism if and only if the surface has only one puncture, so that we have in our context always the condition $n=1$.

We come now to the 4 exceptional cases listed in part (c) of their Theorem 2: The first case $(6 ;-1)$ can not be realized by a pseudo-Anosov map with nonorientable stable foliation, but according to their Theorem 2 there must be a realization by a pseudo-Anosov with orientable stable lamination. The last case, $(;-1)$ requires more than one puncture, so that it is ruled out by the previous paragraph. The third case, $(1,3 ;-1)$, adds up to $g=1$, so that for $n=1$ one has $N=2$ : In this case all automorphisms are geometric, and hence there is no chance to realize the corresponding index list $\left[\frac{1}{2}, \frac{1}{2}\right]$. However, in the paper here we always assume $N \geq 3$, so that this case does not occur.

There remains the second exceptional case: $(3,5 ;-1)$. In this case we have $g=2$. From $n=1$ we deduce the following two possibilities for the index list: $\left[\frac{5}{2}, \frac{1}{2}\right]$ or $\left[\frac{3}{2}, \frac{3}{2}\right]$, according to which of the two singularities coincides with the puncture. The former index list is alternatively realized by the case $(1,7 ;-1)$ from [10] (with the puncture at the singularity with only 1 prong), which satisfies all conditions of Theorem 2 of [10]. The other index list, $\left[\frac{3}{2}, \frac{3}{2}\right]$, however, leads always back to their second exceptional case $(3,5 ;-1)$, and hence can not be realized by a geometric automorphism. There remains as a last "leftover mystery" of the index realization problem for $N \geq 3$ the question whether the index list $\left[\frac{3}{2}, \frac{3}{2}\right]$ can perhaps be realized by a parageometric automorphism of $F_{4}$.

7.3. Some numerical experiments regarding the stable index. Our realization result naturally leads to the question of frequency of the different 
index lists. Thanks to the program developed by the first author in Python and Sage we were able to do the following numerical experiments.

Fix a finite alphabet $A$ with $N$ letters and let $F_{A}$ be the free group on $A$. With Convention 4.2, an elementary Nielsen automorphism is given by $a \mapsto a b$ with $a, b \in A^{ \pm 1}, a \neq b$ and $a \neq b^{-1}$. Recall that elementary Nielsen automorphisms form a generating set of $\operatorname{Aut}\left(F_{A}\right)$ and $\operatorname{Out}\left(F_{A}\right)$.

Our program tries random products of $L$ elementary Nielsen automorphisms, that is to say they approximate the random walk on $\operatorname{Out}\left(F_{A}\right)$ for this generating set.

Each line of the table below corresponds to a sample size of 100 computed random automorphisms. Computations were made at the math department in Marseille, without compiling the Sage code nor looking for serious optimization.

Note that those automorphisms commonly involve words with several thousands of letters. Note also that those frequencies are not completly significant. First, Rivin [12] (see also [13]) proved that the frequency of iwips goes to $100 \%$ when the number of elementary Nielsen automorphisms in the product goes to infinity (but 26 or even 41 are quite small compare to infinity). Different tests with the above data may lead to slightly different results. However, what seems to be significant is that:

(1) Automorphisms with small indices are much more frequent that automorphisms with high indices.

(2) Automorphisms with index greater than half the theoretical maximal $\left(\frac{N-1}{2}\right)$ almost never occur. In particular the maximal index $N-1$ never occured in our tests out of thousands of tries.

(3) Several index lists seem to share positive frequency.

(4) The smallest index list: $\left[\frac{1}{2}\right]$, is not always the most frequent.

We have no clue on how to prove or disprove such experimental observations.

\begin{tabular}{|c|c|c|c|c|c|c|c|c|}
\hline \multirow{3}{*}{$\mathrm{N}$} & \multirow{3}{*}{$\mathrm{L}$} & \multicolumn{6}{|c|}{ Frequency } & \multirow{3}{*}{$\begin{array}{c}\text { Computation } \\
\text { time }\end{array}$} \\
\hline & & \multirow{2}{*}{ iwips } & \multicolumn{5}{|c|}{ most frequent index lists among iwips } & \\
\hline & & & {$\left[\frac{1}{2}\right]$} & {$\left[\frac{1}{2}, \frac{1}{2}\right]$} & [1] & {$\left[\frac{1}{2}, \frac{1}{2}, \frac{1}{2}\right]$} & {$\left[\frac{1}{2}, \frac{1}{2}, \frac{1}{2}, \frac{1}{2}\right]$} & \\
\hline 3 & 26 & $100 \%$ & $64 \%$ & $34 \%$ & $1 \%$ & $0 \%$ & $0 \%$ & $2 \min$ \\
\hline 4 & 26 & $97 \%$ & $47 \%$ & $34 \%$ & $3 \%$ & $14 \%$ & $1 \%$ & $4 \mathrm{~min}$ \\
\hline 5 & 26 & $93 \%$ & $29 \%$ & $32 \%$ & $3 \%$ & $28 \%$ & $5 \%$ & $7 \mathrm{~min}$ \\
\hline 6 & 29 & $95 \%$ & $21 \%$ & $29 \%$ & $6 \%$ & $20 \%$ & $9 \%$ & $15 \min$ \\
\hline 7 & 34 & $91 \%$ & $17 \%$ & $26 \%$ & $9 \%$ & $25 \%$ & $7 \%$ & $23 \mathrm{~min}$ \\
\hline 8 & 36 & $84 \%$ & $13 \%$ & $19 \%$ & $7 \%$ & $17 \%$ & $18 \%$ & $31 \mathrm{~min}$ \\
\hline 9 & 39 & $78 \%$ & $11 \%$ & $6 \%$ & $11 \%$ & $18 \%$ & $10 \%$ & $46 \min$ \\
\hline 10 & 41 & $76 \%$ & $3 \%$ & $8 \%$ & $8 \%$ & $5 \%$ & $8 \%$ & $1 \mathrm{~h} 5 \mathrm{~min}$ \\
\hline
\end{tabular}




\section{REFERENCES}

[1] M. Bestvina, and M. Handel, Train tracks and automorphisms of free groups. Ann. of Math. (2) 135 (1992), no. 1, 1-51

[2] T. Coulbois and A. Hilion, Ergodic currents dual to a real tree, preprint, 2013; arXiv:1302.3766

[3] T. Coulbois, and M. Lustig, Long turns, INP's and index for free group automorphisms. Preprint 2014

[4] D. Gaboriau, A. Jaeger, G. Levitt, and M. Lustig, An index for counting fixed points of automorphisms of free groups. Duke Math. J. 93 (1998), no. 3, 425-452

[5] M. Handel and L. Mosher, Axes in Outer Space, Memoirs AMS 1004, Amer Mathematical Society, 2011

[6] A. Jäger and M. Lustig, Free group automorphisms with many fixed points at infinity. The Zieschang Gedenkschrift, 321-333, Geom. Topol. Monogr., 14, Geom. Topol. Publ., Coventry, 2008

[7] S. Leibman, Stability under powers of minset of hyperbolic irreducible automorphism, PhD Thesis, Utah University, August 2014

[8] M. Lustig, Structure and conjugacy for automorphisms of free groups I. MPI-Bonn Preprint Series 2000, No. 241

[9] M. Lustig, Conjugacy and centralizers for iwip automorphisms of free groups. In "Geometric Group Theory", Trends in Mathematics, 197-224. Birkhäuser Verlag, Basel, 2007

[10] H. Masur and J. Smillie, Quadratic differentials with prescribed singularities and pseudo-Anosov diffeomorphisms. Comment. Math. Helvetici 68 (1993), 289-307

[11] C. Pfaff, Out $\left(F_{3}\right)$ Index realization. arXiv:1311.4490

[12] I. Rivin, Walks on groups, counting reducible matrices, polynomials, and surface and free group automorphisms. Duke Math. J., 142 (2008), 353-379.

[13] A. Sisto, Contracting elements and random walks. arXiv:1112.2666

Institut de mathématiques de Marseille

Université d'Aix-Marseille

39, rue Frédéric Joliot Curie

13453 Marseille Cedex 13

France

thierry.coulbois@univ-amu.fr

martin.lustig@univ-amu.fr

Institut de mathématiques de Marseille, Université d'Aix-Marseille, 39, Rue Frédéric Joliot Curie, 13453 Marseille Cedex 13, France, thierry.coulbois $@$ UNIV-AMU.FR, MARTIN.LUSTIGQUNIV-AMU.FR 